\title{
Gastrointestinal involvement in POEMS syndrome: a novel clinical manifestation
}

\author{
S Dogan, Y Beyazit, A Shorbagi, S Köklü, S Ustunel, N Guler, A Uner
}

Postgrad Med J 2005;81:e12 (http://www.postgradmedi.com/cgi/content/full/81/959/e12). doi: 10.1136/pgmj.2004.02961 1

POEMS syndrome (polyneuropathy, organomegaly, endocrinopathy, monoclonal gammopathy, and skin changes) is characterised by a rare multisystem disorder of unknown pathogenesis. Although its pathophysiology is not well understood, overexpression of proinflammatory cytokines has been implicated. Gastrointestinal system disorders have not been reported among the components of the syndrome. A case is reported of POEMS syndrome with gastrointestinal involvement shown by gastrointestinal endoscopy.

$\mathrm{P}$ OEMS syndrome is a rare multisystem disorder characterised by polyneuropathy, organomegaly (hepatomegaly, lymphadenopathy, or splenomegaly), endocrinopathy (including diabetes, impotence, amenorrhoea, gynaecomastia, hypothyroidism and adrenal failure), monoclonal gammopathy (usually of IgA-lambda), skin changes, and sclerotic bone changes. ${ }^{1}$ Although not represented in the acronym, the syndrome may include other important features such as Castleman's disease, pleural effusion, thrombocytosis, renal dysfunction, oedema, ascites, pulmonary hypertension, and congestive heart failure. ${ }^{2}$ We report on a patient with POEMS syndrome who had gastric and colonic involvement and we discuss this rare concomitance in the light of the relevant literature.

\section{CASE REPORT}

A 37 year old woman was admitted to our hospital with three months' history of polydipsia, polyuria, weight loss $(8 \mathrm{~kg}$ in three months), dyspepsia, and diarrhoea (8-10 times/day), myalgia, bone pain, and progressive weakness of both lower limbs that left the patient chairbound. Her medical history was unremarkable. Physical examination showed mild hepatomegaly, axillary and inguinal lymphadenopathies, generalised skin hyperpigmentation, oedema, and moderate proximal and distal sensory loss. The remaining systemic examination was normal.

Laboratory examination showed a normal whole blood count and erthyrocyte sedimentation rate. Serum transaminases, creatinine, and urea levels were within normal range. Serological testing for Helicobacter pylori was found to be negative. Stool leucocyte, ovum parasite evaluation, and also stool culture was negative. Although fasting plasma glucose level was $23.5 \mathrm{mmol} / \mathrm{l}(3.9-6.1)$, insulin therapy was started on admission. Test for rheumatoid factor, cryoglobulin, antinuclear antibody, anti-double stranded DNA antibody, and antineutrophil cytoplasmic autoantibody were negative. Bence Jones proteins were not detected. Electromyographic findings were consistent with demyelinating peripheral neuropathy. Computed tomograms of the abdomen showed multiple sclerotic bone lesions, hepatomegaly, and intraabdominal lymphadenopathy with no splenomegaly. Portal Doppler ultrasonography was normal. Cranial magnetic resonance imaging showed decreased bone marrow intensity in all the skull bones suggesting myelofibrosis. Immune electrophoresis showed a monoclonal gammopathy (immunoglobulin G lambda), and the serum Ig G level was $15.81 \mathrm{~g} / \mathrm{l}$ (7.51-15.60). Bone marrow histological examination showed diffuse atypical plasma cell infiltration. Inguinal lymph node biopsy showed angiofolliculer hyperplasia with hyaline vascular transformation of the lymphoid follicles, which is characteristic for Castleman's disease.

Gastric endoscopy showed a snakeskin-like appearance of stomach and duodenum (fig 1), and whole colonic mucosa was hyperaemic on colonoscopy. Multiple biopsy specimens from several regions of the colon mucosa were taken. Microscopic examination showed infiltration of lamina propria by numerous inflammatory cells, mainly by neutrophils (fig 2). Amyloid staining was negative.

A diagnosis of POEMS syndrome was reached based on the presence of polyneuropathy, hepatomegaly, endocrinopathy (diabetes mellitus), monoclonal gammopathy, and skin hyperpigmentation. The patient was treated with CHOP protocol including cyclophosphamide $(1100 \mathrm{mg})$ for one day, adriamycin $(75 \mathrm{mg})$ for one day, vincristine $(2 \mathrm{mg})$ for one day, prednisolone $(150 \mathrm{mg})$ for five days. After chemotherapy her myalgia, weakness, skin oedema, and paresthesia showed considerable improvement and also gastrointestinal symptoms including diarrhoea and dyspepsia disappeared. She was discharged 30 days after admission. A repeat upper gastrointestinal endoscopy and colonoscopy was performed 45 days after initial chemotherapy. Biopsy specimens taken both from stomach and colon were completely normal. Thereafter, she received a total of six cycles of CHOP in the outpatient clinic. Twelve months after discharge, the patient remains well except a mild paresthesia on both lower limbs.

\section{DISCUSSION}

POEMS syndrome also known as Crow fucase syndrome, was first described in Japan as a rare multisystem disease

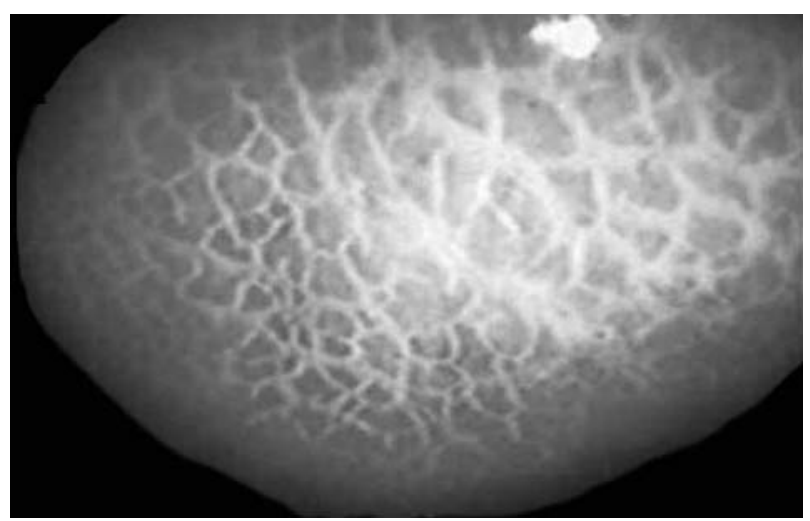

Figure 1 A snakeskin-like appearance of gastric mucosa. 


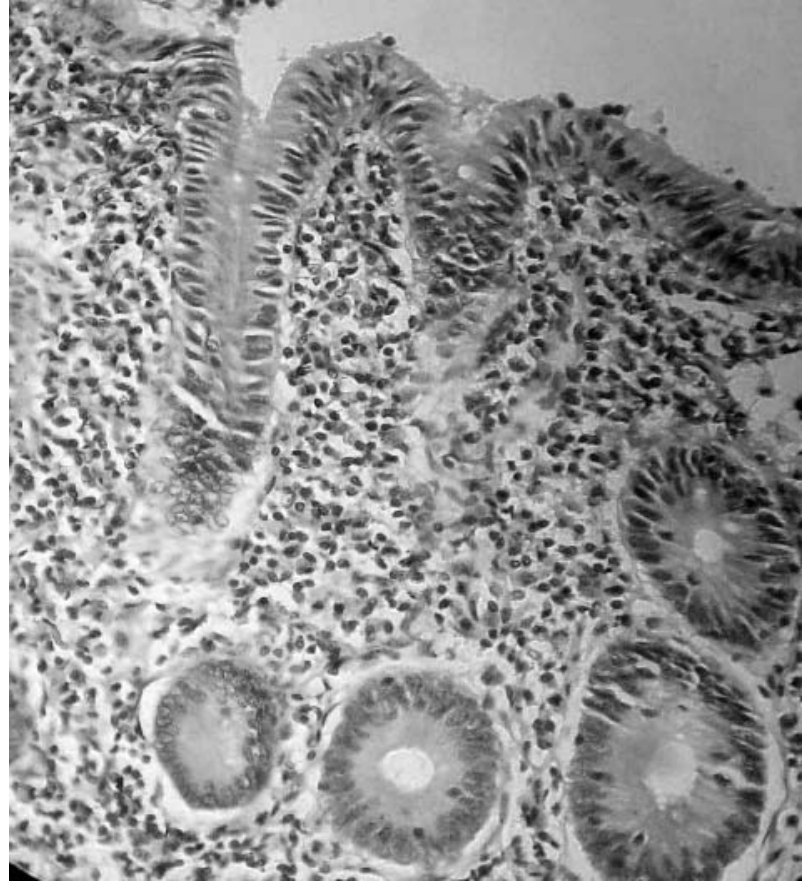

Figure 2 Colonoscopic biopsy specimen that shows neutrophilic infiltration of the lamina propria consistent with colitis.

characterised by polyneuropathy, organomegaly, endocrinopathy, monoclonal gammopathy, and skin lesions. ${ }^{3}$ Our patient fulfilled all of the above mentioned characteristic criterias and the diagnosis of POEMS syndrome was made.

Gastrointestinal involvement is not a part of the syndrome but gastrointestinal symptoms may be an accompanying clinical feature. ${ }^{4}$ There are many cases of POEMS syndrome in the literature but none have suggested gastrointestinal involvement without amyloid. Our patient had snakeskinlike appearance in the upper endoscopy. This form of a gastric lesion has been described as fine white reticular pattern separating areas of pinkish gastric mucosa occurring because of congestion and hyperaemia and is usually seen in portal hypertensive gastropathy. ${ }^{5}$ There was no evidence of portal hypertension and related disorders in our patient, and this appearance is probably related to POEMS syndrome.

The colonoscopic biopsy of our patient was consistent with colitis, and amyloid staining was negative. There is only one reported case in the literature regarding POEMS syndrome associated gastrointestinal symptoms, in which the gastrointestinal involvement was attributed to reactive amyloidosis. ${ }^{4}$

The pathophysiology of POEMS syndrome remains poorly understood. Many patients with POEMS syndrome have high levels of interleukin $1 \beta$, interleukin 6 , and tumour necrosis factor $\alpha .{ }^{67}$ These changes may be responsible for some of the clinical manifestations but they fail to explain the whole clinical picture. Preliminary data suggest that raised vascular endothelial growth factor levels may play an important part in the pathogenesis of POEMS syndrome. ${ }^{8}$ The consequent immune inflammatory response is sustained by $\mathrm{T}$ cell activation and proinflammatory cytokines. Inflammatory cytokines, such as interleukin 1, interleukin 6, and tumour necrosis factor have diverse effects on tissues. They promote fibrogenesis, collagen production, activation of tissue metalloproteinases, and the production of other inflammatory mediators. ${ }^{9}$ Similarly these mechanisms have also been implicated in the pathogenesis of colitis thus we strongly believe that the colitis of our patient could well have been attributable to the immune dysregulation and cytokine induced intestinal epithelial cell apoptosis. ${ }^{2}{ }^{10}$

The prognosis for patients with POEMS has been reported to be poor, with median survivals estimated to be 12 to 165 months. ${ }^{2}{ }^{10} 11$ Survival is not affected by the number of POEMS features. Clubbing, extravascular volume overload, stroke, and myocardial infarction are associated with shorter survival. Because the pathogenesis of this multisystem disease is elusive, treatment is not standardised. Many treatment options have been used, including radiation, plasmapheresis, intravenous immunoglobulin, interferon alfa, corticosteroids, alkylators, azathioprine, autologous stem cell transplantation, tamoxifen, and transretinoic acid. ${ }^{2}$ Alkylators with or without corticosteroids are effective in some patients. Although CHOP is not a standard treatment protocol for POEMS disease, our patient gave a good response to therapy.

Overall we advocate that patients with POEMS syndrome who have gastrointestinal complaints should thoroughly be evaluated for gastrointestinal involvement. We also highlight the necessity of further studies for better delineation of the characteristics of this entity in POEMS syndrome.

\section{Authors' affiliations}

S Dogan, Y Beyazit, A Shorbagi, Department of Internal Medicine, Hacettepe University, Faculty of Medicine, Ankara, Turkey

S Köklü, Department of Gastroenterology, Turkiye Yuksek Ihtisas Hospital, Ankara, Turkey

S Ustunel, Hacettepe University, Faculty of Medicine

N Guler, Department of Medical Oncology, Hacettepe University, Faculty of Medicine

A Uner, Department of Pathology, Hacettepe University, Faculty of Medicine

Funding: none.

Competing interest: none.

Correspondence to: Dr S Köklü, Tıp Fakültesi caddesi, 259/3, Mamak, Ankara, Turkey; gskoklu@ yahoo.com

Submitted 11 October 2004

Accepted 9 March 2005

\section{REFERENCES}

1 Bardwick PA, Zvaifler NJ, Gill GN, et al. Plasma-cell dyscrasia with polyneuropathy, organomegaly, endocrinopathy, Mprotein and skin changes. The POEMS syndrome. Report of two cases and review of the literature. Medicine 1980;59:311-22.

2 Dispenzieri A, Kyle RA, Lacy $M Q$, et al. POEMS syndrome: definitions and long-term outcome. Blood 2003;101:2496-506.

3 Nakanishi T, Sobue I, Toyokura Y, et al. The Crow-Fukase syndrome. A study of 102 cases in Japan. Neurology 1984;34:712-20.

4 Kihara $\mathrm{Y}$, Hori H, Murakami H, et al. A case of POEMS syndrome associated with reactive amyloidosis and Waldenstrom's macroglobulinaemia. J Intern Med 2002;252:255-8.

5 Bernstein DE, Phillips RS. Portal hypertensive gastropathy. Gastrointest Endosc Clin N Am 1996;6:697-708

6 Gherardi RK, Belec L, Fromont G, et al. Elevated levels of interleukin-1 beta (IL1 beta) and IL- 6 in serum and increased production of IL- 1 beta mRNA in lymph nodes of patients with polyneuropathy, organomegaly, endocrinopathy, $M$ protein, and skin changes (POEMS) syndrome. Blood 1994;83:2587-93.

7 Lesprit P, Authier FJ, Gherardi R, et al. Acute arterial obliteration: A new feature of the POEMS syndrome? Medicine 1996;75:226-32.

8 Soubrier M, Sauron C, Souweine B, et al. Growth factors and proinflammatory cytokines in the renal involvement of POEMS syndrome. Am J Kidney Dis 1999;34:633-8.

9 Blumberg RS, Saubermann $\amalg$, Strober W. Animal models of mucosal inflammation and their relation to human inflammatory bowel disease. Curr Opin Immunol 1999;11:648-56.

10 Yamamoto $M$, Yoshizaki K, Kishimoto T, et al. IL-6 is required for the development of Th1 cell-mediated murine colitis. J Immunol 2000; 164:4878-82. 